\title{
Effect of Sequencing Batch Cycle Strategy on the Treatment of a Simulated Textile Wastewater with Aerobic Granular Sludge
}

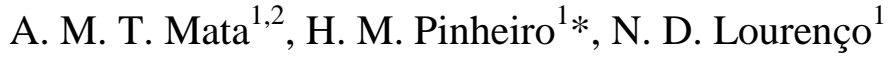 \\ (1) $\mathrm{iBB}$ - Institute for Bioengineering and Biosciences, Instituto Superior Técnico, Departamento \\ de Bioengenharia, Universidade de Lisboa, Av. Rovisco Pais, 1049-001 Lisboa, Portugal \\ (helena.pinheiro@tecnico.ulisboa.pt; nidia.lourenco@ tecnico.ulisboa.pt) ${ }^{(2)}$ Escola Superior de \\ Teconologia de Setúbal do Instituto Politécnico de Setúbal, Rua Vale de Chaves, Campus do IPS, \\ Estefanilha, 2910-761 Setúbal, Portugal (ana.mata@estsetubal.ips.pt) *Corresponding author: H. \\ M. Pinheiro; helena.pinheiro@tecnico.ulisboa.pt, +351218419125
}

\begin{abstract}
Aerobic granular sludge was successfully developed on a simulated textile effluent with an added azo dye, on a non-tubular sequential batch reactor (SBR), with better granulation results than in a dye-free control SBR fed with the same base medium. The overall performance of the treatment was very good, with high color and chemical oxygen demand (COD) removal yields (up to 85\% and $80 \%$, respectively). Operation under two sequencing batch cycle strategies, i.e., with a single anaerobic/aerobic reaction phase or under intermittent aeration, showed that the latter generally improved the treatment performance. Under intermittent aeration, biomass accumulation was induced, with faster settling aggregates, leading to a higher COD removal yield. Although a lower decolorization rate was observed in the first 30 minutes of anaerobic reaction, as compared to the single phase strategy, the overall color removal remained high. This work demonstrates the applicability of the new aerobic granular sludge technology for the treatment of textile effluents.
\end{abstract}

\section{Keywords}

Wastewater Treatment; Biodegradation; Aggregation; Bioreactors; Sequential Batch Reactor; Azo Dye

\section{List of Abbreviations}

2A1N4S - 2-Amino-1-naphthol-4-sulphonate 4A1NS - 4-Amino-1-naphthalene sulphonate 


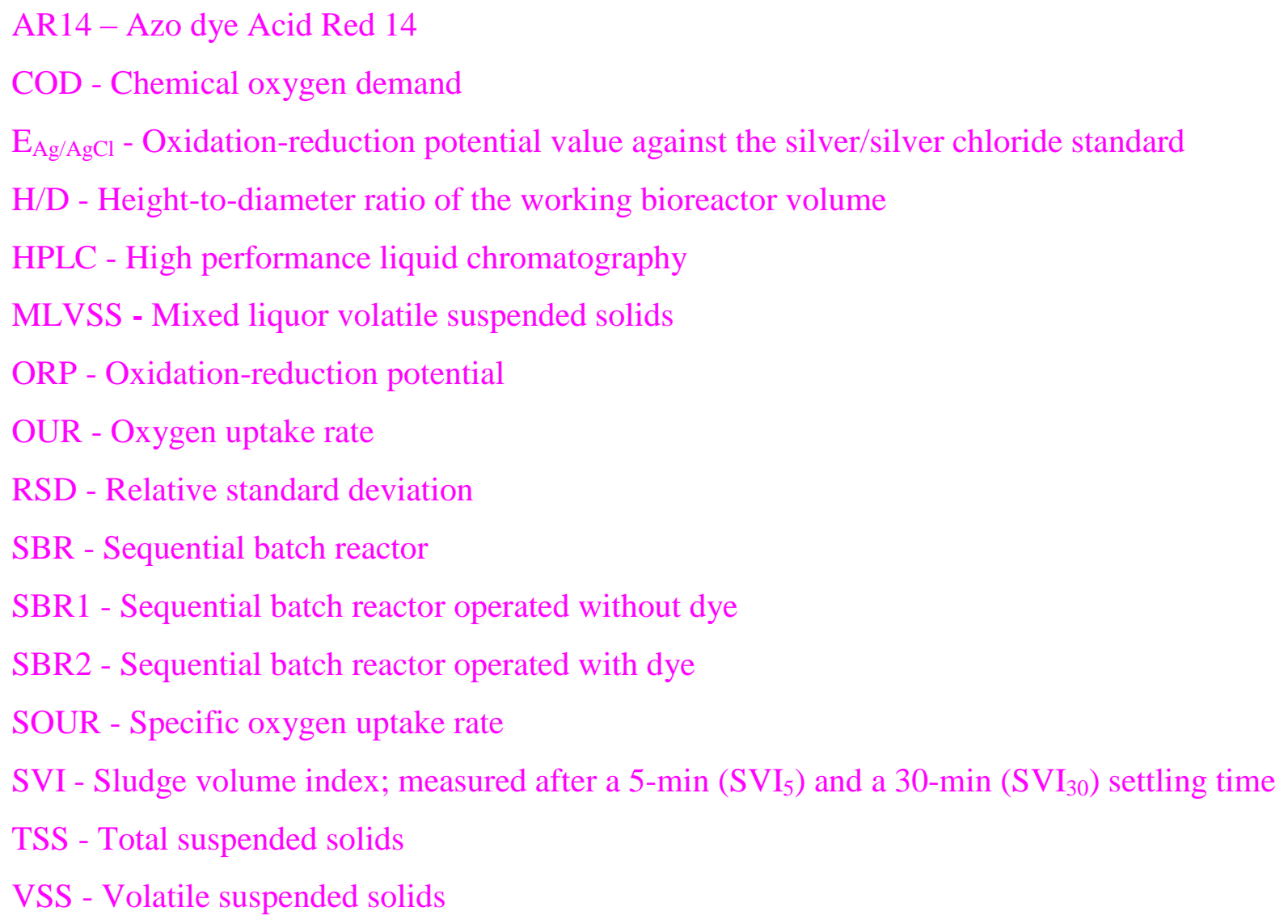

\section{Introduction}

The modern textile industry has been swift to respond to the increased demand for textile products worldwide, and its wastewater load has been increasing proportionally [1]. This situation has sometimes led to the degradation of the quality of receiving water bodies, limiting their use for several purposes [2] . This industrial sector generates large quantities of wastewater, e.g., in Europe, a water consumption of $50-240 \mathrm{~m}^{3}$ per ton of textile product has been estimated [3]. These effluents are usually heavily loaded with organic carbon and color compounds, the latter being azo dyes in an estimated $70 \%$ of the cases [4]. The treatment of textile effluents poses a major challenge in environmental protection, namely in what regards cost and quality of the final effluent. Physicochemical treatments are available but costly, and in spite of notable achievements lately reported on the application of bio-treatments to textile wastewaters [1], the latter are not yet efficient enough notably in what regards the elimination of synthetic colorants. 

of textile effluents, with dye reduction occurring in the anaerobic phase, resulting in decolorization, and further organic load removal being obtained in the subsequent aerobic phase, with possible oxidation of dye reduction metabolites [5-7]. Sequential batch reactors (SBR) have been often reported as good options for this purpose [5, 7] due to their operational flexibility and simplicity, and compact layout [5]. The SBR configuration in activated sludge treatment systems is characterized by cyclic, fill-an-draw operation of a single tank, dispensing the use of dedicated settlers. The standard cycle comprises five steps: fill (influent feed, static or with agitation and/or aeration), react (with agitation and/or aeration), settle (sedimentation of the biomass/clarification of the treated liquor), draw (clarified effluent discharge) and idle [8]. Excess biomass can be withdrawn during the react or draw phases. This system allows the manipulation of reaction and settling times in each cycle, in response to variations in input organic loads and biomass settleability. This feature is particularly useful for wastewaters coming from batchwise industrial processes, such as textile dyeing, which often exhibit wide variations in flow rate and composition [5, 6]. SBR operation also easily allows the implementation of the two-step anaerobic-aerobic reaction sequence required for reductive decolorization and organic load oxidation in dye-containing textile wastewaters $[6,7]$.

Aerobic granular sludge is a new and promising technology for the treatment of industrial wastewaters [9]. Aerobic granules are self-aggregated microorganism clusters with outstanding settling properties, when compared with conventional activated sludge flocs. In the present stateof-the-art they are obtained and maintained only in SBR $[10,11]$ reducing to a few minutes the settling time in these single-tank systems [12]. Very high biomass concentrations can be achieved in the bioreactor, resulting in high organic load treatment capacities and very compact systems [12]. Aerobic and anoxic/anaerobic zones can coexist inside the same granule, in response to the adjustment of aerated and non-aerated periods in the SBR cycle. This provides a unique system for the removal of organic load and nutrients since the major biological reactions can occur simultaneously even throughout the aerated phase, namely, the accumulation of polyphosphates and nitrification in the outer layers and denitrification in the inner granule [10,13,14]. Other advantages of aerobic granules are their robustness in the presence of toxic compounds [15] and the rapid recovery of biological activity and maintenance of granule integrity even after extended storage periods [16-19]. These are especially important in industrial wastewater treatment 


\section{Materials and Methods}

\subsection{Simulated textile effluent feed solutions}

A carbon source stock solution was prepared with a starch-derived sizing agent used in the textile industry, Emsize E1 (hydroxypropyl-starch, Emsland-Starke GmbH, Germany) as the carbon source. This substrate was pre-hydrolyzed in alkaline conditions $(\mathrm{NaOH})$ as described previously [5], followed by $\mathrm{pH}$ adjustment to $7.0(\mathrm{HCl})$ and dilution with deionised water to $100 \mathrm{~g} . \mathrm{L}^{-1}$. A base feed solution was prepared in deionized water using the carbon source stock solution diluted to a chemical oxygen demand (COD) content of $1000 \mathrm{mg} \mathrm{O}_{2} \cdot \mathrm{L}^{-1}$, and supplemented with $\mathrm{pH}$ buffering phosphates and nutrients to the following concentrations: $\mathrm{KH}_{2} \mathrm{PO}_{4}\left(760 \mathrm{mg} . \mathrm{L}^{-1}\right)$, $\mathrm{Na}_{2} \mathrm{HPO}_{4} .12 \mathrm{H}_{2} \mathrm{O}\left(2310 \mathrm{mg} . \mathrm{L}^{-1}\right), \mathrm{NH}_{4} \mathrm{Cl}\left(140 \mathrm{mg} . \mathrm{L}^{-1}\right), \mathrm{MgSO}_{4} .7 \mathrm{H}_{2} \mathrm{O}$ (23 mg.L $\left.{ }^{-1}\right), \mathrm{CaCl}_{2}(28$ $\left.\mathrm{mg} . \mathrm{L}^{-1}\right), \mathrm{FeCl}_{3} \cdot 6 \mathrm{H}_{2} \mathrm{O}\left(250 \mu \mathrm{g} . \mathrm{L}^{-1}\right), \mathrm{MnSO}_{4} \cdot 4 \mathrm{H}_{2} \mathrm{O}\left(40 \mu \mathrm{g} . \mathrm{L}^{-1}\right), \mathrm{H}_{3} \mathrm{BO}_{3}\left(57 \mu \mathrm{g} . \mathrm{L}^{-1}\right), \mathrm{ZnSO}_{4} \cdot 7 \mathrm{H}_{2} \mathrm{O}$ 


\subsection{Non tubular sequencing batch reactor (SBR) configuration}

The experiments were conducted in two non tubular SBR, SBR1 and SBR2, each with a working volume of $1.5 \mathrm{~L}$, operated with a volumetric exchange ratio of $50 \%$, the settled effluent being withdrawn at half-height of the reactor's content, and at a hydraulic retention time value of $12 \mathrm{~h}$. Each reactor had a working height of $25 \mathrm{~cm}$ and an inner diameter of $10 \mathrm{~cm}$, the height-todiameter ratio (H/D) value being 2.5. For aeration an air compressor model SPP 15 (Highblow, Japan) was used with a porous membrane diffuser placed at the bottom of each reactor. The air flow rate was approximately $3.0 \mathrm{~L} \cdot \mathrm{min}^{-1}$ for each reactor, giving an aeration rate of 2 v.v.m. The SBR were operated under mechanical stirring during the entire reaction periods, both aerated and non-aerated. The magnetic stirrer used was a bioMIXdrive 1 model with the bioMIXcontrol MS4 control unit run at 200 r.p.m. (2mag, Germany). The base feed solution was inserted at the bottom of the reactor, at the level of the air diffuser, using a separate peristaltic pump for each reactor (Mini-S 660, Ismatec, Switzerland), and the rapid discharge of the settled supernatant was achieved using a single gear pump for the two reactors (Reglo-Z, Ismatec, Switzerland). A separate peristaltic pump was used to feed the dye stock solution to SBR2. The pumping, aeration and agitation functions were automatically controlled, via an interface, by a dedicated program installed on a personal computer. 


\subsection{Experimental strategy}

The two SBR were operated under the same operational conditions, except for the presence of dye in the feed. In this way, the effect of the dye could be assessed with two independent experiments in all conditions imposed throughout the study. The duration of the operational cycle was kept at 6 hours, totaling 4 cycles per day. Each cycle comprised the following phases (Table 1): fill, during which fresh feed was pumped into the reactor, over the settled biomass leftover from the previous cycle, without mechanical mixing; reaction, during which mechanical mixing was applied, both without and with aeration; settling, without mechanical mixing for biomass sedimentation; discharge, during which half of the operational volume was rapidly removed from the clarified supernatant; and idle, a quiescent period to complete the cycle time. Thus, the reactors' liquid volume was renewed twice each day (cycle exchange ratio: 50\%). The organic loading rate fed to the reactors was kept at $2.0 \mathrm{kgCOD} \cdot \mathrm{m}^{-3} \cdot \mathrm{d}^{-1}$ in all periods.

The experimental strategy included two objectives: 1) following the process of converting the biomass' initial floc morphology to granule morphology (granulation), in the presence and absence of the dye, using an operational cycle which included a prolonged anaerobic phase; 2) testing the system's performance and biomass morphology stability when subjected to changes in the operational cycle.

Within the first objective, all parameters were kept constant except for a progressive reduction in the time allowed for biomass settling before the onset of the supernatant discharge phase (period Ia). At the start of this period the two reactors were seeded with activated sludge flocs (2.7 gTSS.L ${ }^{-1}$ ) harvested at a full-scale wastewater treatment plant (Chelas, Lisbon) and operated during the first 4 days with a settling time of $40 \mathrm{~min}$, for adaptation of the biomass to the synthetic feed. Subsequently, the settling time was shortened to $10 \mathrm{~min}$ (days 5-12), 5 min (days 13-28), and 4 min (days 29-33) to induce granulation [10, 22, 26-29]. Except for the first, all step reductions of the settling time were introduced as soon as the total suspended solids (TSS) level in the effluent was reduced to $100 \mathrm{mg} . \mathrm{L}^{-1}$ or less.

Within the second objective, the duration of the anaerobic phase was first extended (days 34-52), to induce further carbon source storage by the biomass possibly favoring the stability of the granule morphology (period $\mathrm{Ib}$ ). After this, the biomass was collected and used to re-inoculate the reactors under the final operational conditions of period Ia. After a few cycles, an alternative 


\subsection{Analytical Methods}

Bioreactor parameters measured in situ were oxidation-reduction potential (ORP), dissolved oxygen and temperature. All other parameter values were measured on samples collected from the reactors' liquid contents. For $\mathrm{COD}$, dye concentration, $\mathrm{pH}$ and high performance liquid chromatography (HPLC) samples were centrifuged for $10 \mathrm{~min}$ at $4000 \mathrm{rpm}$, and measurements

\begin{tabular}{|c|c|c|c|}
\hline \multirow{2}{*}{ SBR cycle phase duration } & \multicolumn{2}{|c|}{ Period I } & \multirow{2}{*}{ Period II } \\
\hline & Ia & $\mathrm{Ib}$ & \\
\hline Operational time (days) & 1 to 33 & 34 to 52 & 1 to 23 \\
\hline Fill (no mixing) & $15 \mathrm{~min}$ & $15 \mathrm{~min}$ & $15 \mathrm{~min}$ \\
\hline Anaerobic phase (with mixing) & $3 \mathrm{~h}$ & $3.5 \mathrm{~h}$ & \multirow{2}{*}{$\begin{array}{l}\text { Intermittent aeration: } 6 \\
\times(30 \mathrm{~min} \text { anaerobic }+ \\
20 \mathrm{~min} \text { aerated })\end{array}$} \\
\hline Aerobic phase (with mixing and aeration) & $2 \mathrm{~h}$ & $2 \mathrm{~h}$ & \\
\hline Settling & 40 to $4 \mathrm{~min}$ & $4 \min$ & $3 \min$ \\
\hline Discharge & $1 \mathrm{~min}$ & $1 \mathrm{~min}$ & $1 \mathrm{~min}$ \\
\hline Idle & 4 to $40 \mathrm{~min}$ & $10 \mathrm{~min}$ & $41 \mathrm{~min}$ \\
\hline Reaction time / Total cycle time & $5 \mathrm{~h} / 6 \mathrm{~h}$ & $5.5 \mathrm{~h} / 6 \mathrm{~h}$ & $5 \mathrm{~h} / 6 \mathrm{~h}$ \\
\hline
\end{tabular}
Throughout the two periods, the reactors were monitored with respect to biomass inventory, morphology and activity (oxygen uptake rate), pollutant conversion (dye and COD), and chemical parameters (oxidation-reduction potential, $\mathrm{pH}$ ) indirectly related to biological activity. Each imposed operational conditions were kept for at least 4 days, completing at least 8 liquid renovations in the bioreactors.

Table 1. Operational cycle phase durations for the two SBR during the successive experimental periods. 

(APHA, 1998). Each sample was measured in duplicate and mean values are presented (relative standard deviation, RSD: average 1.4\%, maximum 6.0\%). The contribution of the azo dye to the COD level was measured in a deionized water solution at $20 \mathrm{mg} / \mathrm{L}$ and found to be negligible, below the detection limit of the method $\left(\leq 10 \mathrm{mgO}_{2} \cdot \mathrm{L}^{-1}\right)$.

Total suspended solids (TSS) and volatile suspended solids (VSS) were measured on samples from the mixed liquor and from the settled effluent, using standard methods (APHA, 1998). Each sample was measured in duplicate and mean values are presented (RSD: average 2.5\%, maximum $21 \%)$.

Sludge volume index (SVI) was determined by measuring the volume occupied by the sludge settled from $1 \mathrm{~L}$ of mixed liquor, after $5\left(\mathrm{SVI}_{5}\right)$ and $30\left(\mathrm{SVI}_{30}\right)$ minutes of settling, and dividing it by the mixed liquor TSS value. This measurement was carried out offline in an Imhoff cone, and the mixed liquor sample was promptly returned to the reactor.

The concentration of azo dye was determined spectrophotometrically, by reading the absorbance of samples at $515 \mathrm{~nm}$ (the wavelength of maximum absorbance in the visible range) using a Specord 200 spectrophotometer (Analytik Jena, Germany). Each sample was measured in duplicate and mean values are presented (RSD: average 0,2\%, maximum 2,4\%).

HPLC analyses were performed using a Merck-Hitachi (Germany) system comprising a L-6200A Intelligent Pump, a L-4250 UV-Vis Detector and a L-7200 Autosampler with a D7000 Interface for computer control, fitted with a reversed phase RP-18, LiChroCART 250-4 column (Merck, Germany), with spectrophotometric detection at $220 \mathrm{~nm}$. The mobile phase, fed at $0.7 \mathrm{~mL} / \mathrm{min}$, was composed of sodium phosphate buffer $(25 \mathrm{mM}, \mathrm{pH}=5.5)$ and acetonitrile, run on a 30-min linear gradient from 100:0 to 50:50 (v/v), followed by a 5-min linear gradient up to 15:85 (v/v) and ending with a step back to 100:0, kept for a further 5-min period. Standard solutions of the dye Acid Red 14 and of the amine 4-amino-1-naphthalene sulphonate (Sigma, USA) in distilled water were run for their respective peak identification and concentration calibration against peak area. Each sample was measured in duplicate and mean values are presented (RSD: average $0.1 \%$, maximum $0.9 \%$ )

Biomass samples from both reactors were observed at magnifications of $40 \times$ and $100 \times$, under a transmission light microscope (BA200, Motic, China) fitted with a digital camera and respective software (Moticam 1000, Motic, China). 

electrode connected to a Metrohm 691 potentiometer (Switzerland).

Oxidation-Reduction Potential (ORP) was measured in situ, using a SenTix ORP electrode (platinum with silver/silver chloride as reference electrode, $3 \mathrm{M} \mathrm{KCl}$ ) (WTW, Germany) connected to a digital $\mathrm{pH}$ meter ( $\mathrm{pH} 538$, WTW, Germany). Measured values are presented as read. Conversion to values relative to the standard hydrogen electrode $\left(E_{h}\right)$ can be done by adding $+207 \mathrm{mV}$ to the readings.

Dissolved oxygen was measured in situ, using a LDO101 Standard Luminescent Dissolved Oxygen Probe (Hach, USA) connected to a multi parameter meter HQ40d (Hach, USA) fitted with a temperature probe. Oxygen uptake rate (OUR) measurements were taken during the aerated reaction phase, by briefly (up to $5 \mathrm{~min}$ ) interrupting aeration and registering the evolution of dissolved oxygen values in time. For this, the probe was immersed in the mixed liquor, at its surface, to a depth of approximately $3 \mathrm{~cm}$.

\section{Results and Discussion}

\subsection{Biomass concentration, settling properties and morphology}

Figure 1 shows the evolution of the TSS values in the mixed liquor. During the first part of period Ia biomass concentration levels were maintained in SBR1 (no dye) and increased in SBR2 (with dye) in spite of the consecutive shortening of the settling period with consequent biomass washout. This strategy of settling time reduction to induce granulation was successful, with $\mathrm{SVI}_{30}$ in SBR2 (with dye) dropping sharply from an initial value of 325 to $67 \mathrm{~mL}^{-1}{ }^{-1}$ on day 27 (Fig.2, Ia). This result indicated an increase in aggregate cohesion [30] with a correspondent alteration in biomass morphology. Microscopic examination (Fig. 3) showed that granules could be observed from day 21 on, in both reactors, with well defined, round or oval shapes, reaching $0.5 \mathrm{~mm}$ in diameter on day 30 in SBR2. Granules showed much less defined outlines in SBR1, where they became enveloped in a diffuse coating of exopolymeric material (filamentous bacteria were apparently not involved) towards the end of period Ia. This observation in SBR1 followed the reduction of the settling time from 5 to 4 min which also resulted in a sharp increase in SVI still in period Ia. This granule deterioration trend was aggravated in period $\mathrm{Ib}$ with the extension of 
the anaerobic phase as seen in figures 2 and 3. Mass balances to the TSS in the reactors gave average sludge age values of 5 days for SBR1 and 12 days for SBR2 in period I, reinforcing that granulation proceeded better in SBR2. In period Ib granules kept their size and shape definition in SBR2, as opposed to those of SBR1, and their abundance even increased. Some granules were observed which were of a much lighter color than the rest, which probably were composed essentially of exopolymeric material.

The period taken for granulation to be achieved in this study is similar to the ranges reported in the literature $[14,31]$ for anaerobic/aerobic granular systems treating urban wastewaters and synthetic effluents in tubular SBR. However, the granule size obtained, up to $0.5 \mathrm{~mm}$, is lower than most of the literature values. This could have been be partly due to the non-tubular geometry of the reactors, leading to a lower selective pressure for granule development, since granules obtained in a sequential batch air-lift bioreactor with a H/D value of 3 showed also a small size of $0.78 \mathrm{~mm}[32]$. The use of mechanical stirring could have also contributed to the granules' reduced size through shear stress [13]. It was previously reported that granules obtained in a SBR with a H/D value of 5.5, operated under the same stirring rate value of the present study (200 $\mathrm{rpm}$ ), though initially larger in size evolved to a uniform size range of 0.6 to $0.8 \mathrm{~mm}$ [33].

In period II, the intermittent aeration regimen apparently reduced the accumulation of the diffuse exopolymeric material in SBR1, resulting in denser clusters (Fig. 3, II), but at same time some filamentous growth was revealed. Nevertheless, the $\mathrm{SVI}_{30}$ values improved, becoming similar to those obtained in period Ia before day 30. The average sludge age value was 6 days. In this period, SBR2 also performed better, the intermittent aeration leading to biomass accumulation above 5.0 gTSS.L ${ }^{-1}$, and faster biomass settling with $\mathrm{SVI}_{30}$ values reaching their best $\left(55 \mathrm{~mL} \cdot \mathrm{g}^{-1}\right)$. Still, full granulation could not be achieved, since the $\mathrm{SVI}_{5}$ and $\mathrm{SVI}_{30}$ did not attain comparable values, a distinctive characteristic of granular biomass [34]. Dense aggregates were observed (Fig.3, II) of a size similar to that of granules in period I, but with a different, less regular shape. The average sludge age rose in SBR2 up to 15 days, denoting an improvement in system stability. In both reactors, the settled effluent TSS levels were generally around $200 \mathrm{mg} \cdot \mathrm{L}^{-1}$. In both reactors, the overall better performance in period II, as compared to period Ib, could have been due to the intermittent exposure of the granules to increased shear from the air flow, as opposed to a 3.5-hour long initial period without this effect (the anaerobic reaction phase). Shear has been reported to favor aerobic granulation $[10,11,27,35,36]$. The biomass concentration 
levels and $\mathrm{SVI}_{30}$ values are in the same range of those obtained by other authors treating a textile wastewater with aerobic granules in a tubular SBR with a H/D value of 12.5 [21]. This result is remarkable, and proves the feasibility of a non-tubular SBR for aerobic granulation on a synthetic dye containing feed.

\subsection{Carbon source transformation, oxygen uptake and $\mathrm{pH}$ changes}

Representative examples of the profile of residual COD along the SBR cycle reaction time are shown in figure 4. During period I the sharpest drop in COD levels consistently occurred following the onset of aeration. COD removal yields in the anaerobic phase were consistently higher in SBR2 (up to 30\%) than in SBR1 (less than 20\%), with overall removal yields around $70 \%$. A high removal yield of organic substrate during the anaerobic phase, preferably $50 \%$ or more [37], has been shown to be determinant for efficient granulation. This effect possibly contributed to a better granulation process in SBR2. In period II, the cumulative COD removal yield during the anaerobic periods improved slightly, to 23\%, in SBR1 and reached 30\% in SBR2, with the overall COD removal yield also increasing in both SBR, reaching $80 \%$. These overall COD removal levels were comparable to those observed for the same carbon source in a two-phase, 24-hour cycle SBR with flocculent activated sludge [38]. In Figure 4 it can be seen that the residual COD reaches the low concentration levels much sooner in period II than in period I, for both SBR. Several authors consider that exposure of the biomass to a feast/famine regimen promotes aerobic granulation [26, 35, 37, 39-42]. A prolonged famine stage, in particular, is important for granule stability [24] and it should take up about $2 / 3$ of the cycle time [36]. This effect is also a possible explanation for the better granulation obtained in period II, with the apparent consumption of the diffuse exopolymeric material, the lowest SVI values of the experiment and the highest biomass accumulation.

Oxygen uptake rate (OUR) was measured at the start and end of the aeration phases during period I, and at the start of the $1^{\text {st }}$ and end of the $6^{\text {th }}$ aeration phases during period II. Average values of the specific OUR readings (SOUR), the OUR divided by the mixed liquor VSS value (MLVSS), are presented in Table 2. Initial SOUR values for period Ia $\left(68-69 \mathrm{mgO}_{2} \cdot \mathrm{gMLVSS}^{-1} \cdot \mathrm{h}^{-1}\right)$ matched the values reported by other authors for aerobic granular sludge, namely values of 69 and 56 $\mathrm{mgO}_{2} \cdot \mathrm{gMLVSS}^{-1} \cdot \mathrm{h}^{-1}$ for glucose and acetate feeds, respectively [28], and of $51 \mathrm{mgO}_{2} \cdot \mathrm{gMLVSS}^{-}$ 
As exemplified in Figure 5, a consistent acidification profile was detected along the anaerobic phase, which was, also consistently, reversed very quickly upon the onset of aeration. This happened in spite of the buffering effect of the phosphate salts in the medium. Differences between SBR1 and SBR2 were not very significant, typically as shown in Figure 5. This result is probably due to the production and subsequent consumption of free volatile fatty acids, indicating the ready fermentation of the carbohydrate carbon source by the biomass. Thus, reducing equivalents required for dye decolorization are apparently readily available. 


\subsection{Dye decolorization}

Color removal yields measured spectrophotometrically in SBR2 for the overall cycle remained stable in the $80-85 \%$ range along the entire experimental run, with a good yield even on the first day of operation, and best by a small margin in period II under intermittent aeration, as it can be seen in Figure 6. These anaerobic biodecolorization yields are in accordance with those of previous reports for the same dye, Acid Red 14 [43-45].

Also, color removal occurred almost solely in the anaerobic reaction phase. During period II it was clear that the onset of aeration after the first $30 \mathrm{~min}$ of anaerobic reaction interrupts decolorization, the latter being resumed only in the following anaerobic phase (Fig.s 6 and 7a). Moreover, in period I most of the final color removal yield was achieved during the first $30 \mathrm{~min}$ of anaerobic reaction, but in the intermittent aeration regime (period II), color removal yields in the first 30-min anaerobic phase dropped to about half, the yield values of period I being attained only after the second anaerobic phase.

Nevertheless, these results together with those discussed in sections 3.1 and 3.2, indicate that in the two phase regimen of period I, the anaerobic phase could best be much shortened. Decolorization yields could probably be maintaining in the same range, prolonging the aerobic phase, which would increase both the exposure to shear stress and the famine period, possibly improving the granulation and COD removal performance of both reactors.

It should be noted that color readings using spectrophotometry at $515 \mathrm{~nm}$ do not entirely reflect residual dye concentration, since some color development (brownish) was noted after the initial reduction. This is probably due to reactions involving the dye reduction metabolites, namely the unstable ortho 2-amino-1-naphthol-4-sulphonate (2A1N4S). Aromatic amines have been shown to undergo auto-oxidation in the presence of oxygen with the resulting products forming colored oligomeric structures [46-48]. Examples of HPLC chromatograms and analytical results are presented in Figure 7 and indicate that less than $1 \%$ of the initial dye content was present at the end of the 3-hour anaerobic phase in period I, and at the end of the overall reaction phase in period II, in contrast with the circa $80 \%$ color removal yields measured spectrophotometrically on aliquots from the same samples. HPLC results (Fig. 7a) confirm the azo bond cleavage stoichiometry, with the dye reduction forming the corresponding amount of the stable amine 4amino-1-naphthalene sulphonate (4A1NS), also indicating that this metabolite was not 


\subsection{Oxidation-reduction potential profiles}

Typical ORP monitoring cycles in the two SBR are presented in Figure 8. Despite the consistent differences observed in the ORP profiles from the two SBR, the measurements of dissolved oxygen concentration in the mixed liquors gave similar results, i.e., consistently under $0.05 \mathrm{mg}$ $\mathrm{O}_{2} \cdot \mathrm{L}^{-1}$ during the anaerobic phase and around $7 \mathrm{mg} \mathrm{O}_{2} \cdot \mathrm{L}^{-1}$ during the aerated phase. Interesting to note is the shoulder in the ORP profile after the onset of aeration, more pronounced in SBR2, suggesting the presence of accumulated reduced substances produced in anaerobic phase being readily oxidized. The subsequent depletion of these reduced substances would account for the second rise in ORP (end of the shoulder). This shoulder pattern is also found in the results of Muda et al. [21] for dissolved oxygen concentration profiles in an anaerobic/aerobic granular sludge SBR treating a synthetic wastewater containing a defined dye mixture. The absence of this shoulder, in the profiles of both reactors during the $1^{\text {st }}$ aeration stage of period II could possibly explain the lower SOUR values measured. 
SBR1 displayed a slower ORP reduction during the anaerobic phase than SBR2, the latter starting this phase with ORP readings already around $-300 \mathrm{mV}$ (coming from the static fill phase). On the other hand, SBR2 readings tended to be stable in the -300 to $-350 \mathrm{mV}$ range in the anaerobic phase regardless of the operational regimen (Ia, Ib or II), while ORP values in SBR1 kept decreasing throughout the anaerobic phase in period I, and drifted upwards along the anaerobic stages of period II (Fig. 8). This result indicates that the reduced decolorization yield in the first anaerobic stage of period II (half of that obtained in period I after $30 \mathrm{~min}$ of anaerobiosis) cannot be explained by different (higher) ORP values. Thus, it is probably related to a biological response of the active biomass to the different environmental conditions created by the intermittent aeration regimen. Still regarding ORP, it is interesting to refer that a supernatant sample (without biomass) collected from SBR2 after the anaerobic reaction of one of the cycles in period I, gave similar ORP readings while still in the absence of aeration $(-300 \mathrm{mV})$ in a separate vessel, but when subjected to aeration increased its $\mathrm{ORP}$ value to $\mathrm{E}_{\mathrm{Ag} / \mathrm{AgCl}}=0 \mathrm{mV}$. This is much higher than the value attained by the rest of the mixed liquor in the presence of biomass in the subsequent aerated phase and is close to the values attained in SBR1, indicating that the redox equilibrium in the presence of oxygen and dye reduction metabolites is biologically mediated. The involvement of the metabolites from the reduction of AR14 in reversible redox processes was also reported by Liu et al. [43]. It is conceivable that the better granulation obtained in SBR2, with higher COD removal yields in the anaerobic phase, is related to the dye acting as an electron acceptor and allowing for increased metabolic energy yields, even if the redox equivalents involved are in small amounts The reversible redox action of the produced metabolites could enhance this process, possibility through the shuttling of electrons with the consequent increase in reaction rates.

\section{Conclusions}

Aerobic granular sludge was successfully developed on a simulated textile effluent in the presence of an azo dye, with better overall granulation results than in its absence, as observed through the operation of the dye-free control SBR1 on the same base feed. The overall performance of the treatment was very good, with high color and COD removal yields. 


\section{Acknowledgements}

This work was financed by Fundação para a Ciência e a Tecnologia (FCT, Portugal) through the BIOTEXTILE project (PTDC/EBB-EBI/120624/2010). AMT Mata and ND Lourenço acknowledge the financial support from FCT, through a doctoral grant (SFRH/BD/49432/2009) and a post-doctoral research grant (SFRH/BPD/88095/2012), respectively. Instituto Politécnico de Setúbal is also acknowledged for the financial support for AMT Mata’s doctoral work.

\section{References}

[1] A.B. dos Santos, F.J. Cervantes, J.B. van Lier, Review paper on current technologies for decolourisation of textile wastewaters: perspectives for anaerobic biotechnology, Bioresour. Technol. 98 (2007) 2369-2385.

[2] C. O’Neill, F.R. Hawkes, D.L. Hawkes, N.D. Lourenço, H.M. Pinheiro, W. Delée, Colour in textile effluents - sources, measurement, discharge consents and simulation: a review, J. Chem. Technol. Biotechnol. 74 (1999) 1009-1018. 
[3] EWA, Efficient use of water in the textile finishing industry, E-WAter, Official Publication of the European Water Association, 2005.

[4] R. Maas, S. Chaudhari, Adsorption and biological decolourization of azo dye Reactive Red 2 in semicontinuous anaerobic reactors, Process Biochem. 40 (2005) 699-705.

[5] N.D. Lourenço, J.M. Novais, H.M. Pinheiro, Effect of some operational parameters on textile dye biodegradation in a sequential batch reactor, J. Biotechnol. 89 (2001) 163-174.

[6] F.P. van der Zee, S. Villaverde, Combined anaerobic-aerobic treatment of azo dyes-A short review of bioreactor studies, Water Res. 39 (2005) 1425-1440.

[7] G. Farabegoli, A. Chiavola, E. Rolle, M. Naso, Decolorization of Reactive Red 195 by a mixed culture in an alternating anaerobic-aerobic Sequencing Batch Reactor, Biochem. Eng. J. 52 (2010) 220-226.

[8] I. Metcalf \& Eddy, Wastewater Engineering, Treatment and Reuse, Fourth edition ed., McGraw-Hill, 2003.

[9] Y. Liu, Wastewater purification: aerobic granulation in sequenching batch reactors, CRC Press, Taylor and Francis Group, Boca Raton, London, New York, 2008.

[10] M.K. de Kreuk, N. Kishida, M.C.M. van Loosdrecht, Aerobic Granular sludge - state of the art, Water Sci. Technol. 55 (2007) 75-81.

[11] Y. Liu, J.H. Tay, State of the art of biogranulation technology for wastewater treatment, Biotechnol. Adv. 22 (2004) 533-563.

[12] L.M. de Bruin, M.K. de Kreuk, H.F. van der Roest, C. Uijterlinde, M.C. van Loosdrecht, Aerobic granular sludge technology: an alternative to activated sludge?, Water Sci. Technol. 49 (2004) 1-7.

[13] M.K. de Kreuk, M.C. van Loosdrecht, Selection of slow growing organisms as a means for improving aerobic granular sludge stability, Water Sci. Technol. 49 (2004) 9-17.

[14] M.K. de Kreuk, J.J. Heijnen, M.C. van Loosdrecht, Simultaneous COD, nitrogen, and phosphate removal by aerobic granular sludge, Biotechnol. Bioeng. 90 (2005) 761-769.

[15] A.M. Maszenan, Y. Liu, W.J. Ng, Bioremediation of wastewaters with recalcitrant organic compounds and metals by aerobic granules, Biotechnol. Adv. 29 (2011) 111-123.

[16] J. Zhu, P.A. Wilderer, Effect of extended idle conditions on structure and activity of granular activated sludge, Water Res. 37 (2003) 2013-2018.

[17] P. Zeng, W.Q. Zhuang, S.T. Tay, J.H. Tay, The influence of storage on the morphology and physiology of phthalic acid-degrading aerobic granules, Chemosphere 69 (2007) 1751-1757.

[18] X. Wang, H. Zhang, F. Yang, Y. Wang, M. Gao, Long-term storage and subsequent reactivation of aerobic granules, Bioresour. Technol. 99 (2008) 8304-8309.

[19] C. Wan, Q. Zhang, D.-J. Lee, Y. Wang, J. Li, Long-term storage of aerobic granules in liquid media: Viable but non-culturable status, Bioresour. Technol. 166 (2014) 464-470.

[20] L. Liu, G.-P. Sheng, W.-W. Li, Z.-H. Tong, R.J. Zeng, J.-X. Liu, J. Xie, S.-C. Peng, H.-Q. $\mathrm{Yu}$, Cultivation of aerobic granular sludge with a mixed wastewater rich in toxic organics, Biochem. Eng. J. 57 (2011) 7-12.

[21] K. Muda, A. Aris, M.R. Salim, Z. Ibrahim, A. Yahya, M.C. van Loosdrecht, A. Ahmad, M.Z. Nawahwi, Development of granular sludge for textile wastewater treatment, Water Res. 44 (2010) 4341-4350.

[22] J.J. Beun, A. Hendriks, M.C.M. van Loosdrecht, E. Morgenroth, P.A. Wilderer, J.J. Heijnen, Aerobic granulation in a sequencing batch reactor, Water Res. 33 (1999) 2283-2290.

[23] S.T. Tay, B.Y. Moy, H.L. Jiang, J.H. Tay, Rapid cultivation of stable aerobic phenoldegrading granules using acetate-fed granules as microbial seed, J. Biotechnol. 115 (2005) 387395. 

formation of aerobic granules, Appl. Microbiol. Biotechnol. 76 (2007) 211-216.

[25] J.P. Bassin, R. Kleerebezem, M. Dezotti, M.C. van Loosdrecht, Measuring biomass specific ammonium, nitrite and phosphate uptake rates in aerobic granular sludge, Chemosphere 89 (2012) 1161-1168.

[26] E. Morgenroth, T. Sherden, M.C.M. Van Loosdrecht, J.J. Heijnen, P.A. Wilderer, Aerobic granular sludge in a sequencing batch reactor, Water Res. 31 (1997) 3191-3194.

[27] J.J. Heijnen, M.C.M. van Loosdrecht, Method for acquiring grain-shaped growth of a microorganismin a reactor, World Intellectual Property Organization, 1998.

[28] J.H. Tay, Q.S. Liu, Y. Liu, Microscopic observation of aerobic granulation in sequential aerobic sludge blanket reactor, J. Appl. Microbiol. 91 (2001) 168-175.

[29] Y. Liu, H.H.P. Fang, Influences of Extracellular Polymeric Substances (EPS) on Flocculation, Settling, and Dewatering of Activated Sludge, Crit. Rev. Environ. Sci. Technol. 33 (2003) 237-273.

[30] J. Wan, I. Mozo, A. Filali, A. Liné, Y. Bessière, M. Spérandio, Evolution of bioaggregate strength during aerobic granular sludge formation, Biochem. Eng. J. 58-59 (2011) 69-78.

[31] M.K. de Kreuk, M.C. van Loosdrecht, Formation of Aerobic Granules with Domestic Sewage, J. Environ. Eng. 132 (2006) 694-697.

[32] L. Zhu, X. Xu, W. Luo, Z. Tian, H. Lin, N. Zhang, A comparative study on the formation and characterization of aerobic 4-chloroaniline-degrading granules in SBR and SABR, Appl. Microbiol. Biotechnol. 79 (2008) 867-874.

[33] M. Verawaty, S. Tait, M. Pijuan, Z. Yuan, P.L. Bond, Breakage and growth towards a stable aerobic granule size during the treatment of wastewater, Water Res. 47 (2013) 5338-5349.

[34] N. Schwarzenbeck, R. Erley, P.A. Wilderer, Aerobic granular sludge in an SBR-system treating wastewater rich in particulate matter, Water Sci. Technol. 49 (2004) 41-46.

[35] J.H. Tay, Q.S. Liu, Y. Liu, The role of cellular polysaccharides in the formation and stability of aerobic granules, Lett. Appl. Microbiol. 33 (2001) 222-226.

[36] S. López-Palau, A. Pinto, N. Basset, J. Dosta, J. Mata-Álvarez, ORP slope and feast-famine strategy as the basis of the control of a granular sequencing batch reactor treating winery wastewater, Biochem. Eng. J. 68 (2012) 190-198.

[37] M.C. Van Loosdrecht, M.K. de Kreuk, Method for the treatment of waste water with sludge granules, World Intellectual Property Organization, 2006.

[38] N.D. Lourenço, J.M. Novais, H.M. Pinheiro, Kinetic Studies of Reactive Azo Dye Decolorization in Anaerobic/aerobic Sequencing Batch Reactors, Biotechnol. Lett. 28 (2006) 733-739.

[39] J.J. Beun, E.V. Verhoef, M.C.M. Van Loosdrecht, J.J. Heijnen, Stoichiometry and kinetics of poly- $\beta$-hydroxybutyrate metabolism under denitrifying conditions in activated sludge cultures, Biotechnol. Bioeng. 68 (2000) 496-507.

[40] J.J. Beun, J.J. Heijnen, M.C.M. van Loosdrecht, N-Removal in a granular sludge sequencing batch airlift reactor, Biotechnol. Bioeng. 75 (2001) 82-92.

[41] B.S. McSwain, R.L. Irvine, P.A. Wilderer, The effect of intermittent feeding on aerobic granule structure, Water Sci. Technol. 49 (2004) 19-25.

[42] M.K. de Kreuk, M. Pronk, M.C.M. van Loosdrecht, Formation of aerobic granules and conversion processes in an aerobic granular sludge reactor at moderate and low temperatures, Water Res. 39 (2005) 4476-4484. 
[43] G. Liu, J. Wang, H. Lu, R. Jin, J. Zhou, L. Zhang, Effects of reduction products of orthohydroxyl substituted azo dyes on biodecolorization of azo dyes, J. Hazard. Mater. 171 (2009) 222-229.

[44] F.P. van der Zee, G. Lettinga, J.A. Field, Azo dye decolourisation by anaerobic granular sludge, Chemosphere 44 (2001) 1169-1176.

[45] S. Seshadri, P.L. Bishop, A.M. Agha, Anaerobic/aerobic treatment of selected azo dyes in wastewater, Waste Manag. 14 (1994) 127-137.

[46] J.A. Field, A.J. Stams, M. Kato, G. Schraa, Enhanced biodegradation of aromatic pollutants in cocultures of anaerobic and aerobic bacterial consortia, Antonie van Leeuwenhoek, 67 (1995) 47-77.

[47] M. Kudlich, M.J. Hetheridge, H.-J. Knackmuss, A. Stolz, Autoxidation Reactions of Different Aromatic o-Aminohydroxynaphthalenes That Are Formed during the Anaerobic Reduction of Sulfonated Azo Dyes, Environ. Sci. Technol. 33 (1999) 896-901. [48] M. Kudlich, A. Keck, J. Klein, A. Stolz, Localization of the Enzyme System Involved in Anaerobic Reduction of Azo Dyes by Sphingomonas sp. Strain BN6 and Effect of Artificial Redox Mediators on the Rate of Azo Dye Reduction, Appl. Environ. Microbiol. 63 (1997) 36913694.

[49] A. Pandey, P. Singh, L. Iyengar, Bacterial decolorization and degradation of azo dyes, Int. Biodeterior. Biodegradation 59 (2007) 73-84.

[50] H.M. Pinheiro, E. Touraud, O. Thomas, Aromatic amines from azo dye reduction: status review with emphasis on direct UV spectrophotometric detection in textile industry wastewaters, Dyes Pigm. 61 (2004) 121-139.

[51] N.C. Tan, A. van Leeuwen, E.M. van Voorthuizen, P. Slenders, F.X. Prenafeta-Boldu, H. Temmink, G. Lettinga, J.A. Field, Fate and biodegradability of sulfonated aromatic amines, Biodegradation, 16 (2005) 527-537.

[52] Y. Hong, J. Guo, Z. Xu, C. Mo, M. Xu, G. Sun, Reduction and partial degradation mechanisms of naphthylaminesulfonic azo dye amaranth by Shewanella decolorationis S12, Appl. Microbiol. Biotechnol. 75 (2007) 647-654.

[53] A. Gottlieb, C. Shaw, A. Smith, A. Wheatley, S. Forsythe, The toxicity of textile reactive azo dyes after hydrolysis and decolourisation, J. Biotechnol. 101 (2003) 49-56. 


\section{FIGURE 1}
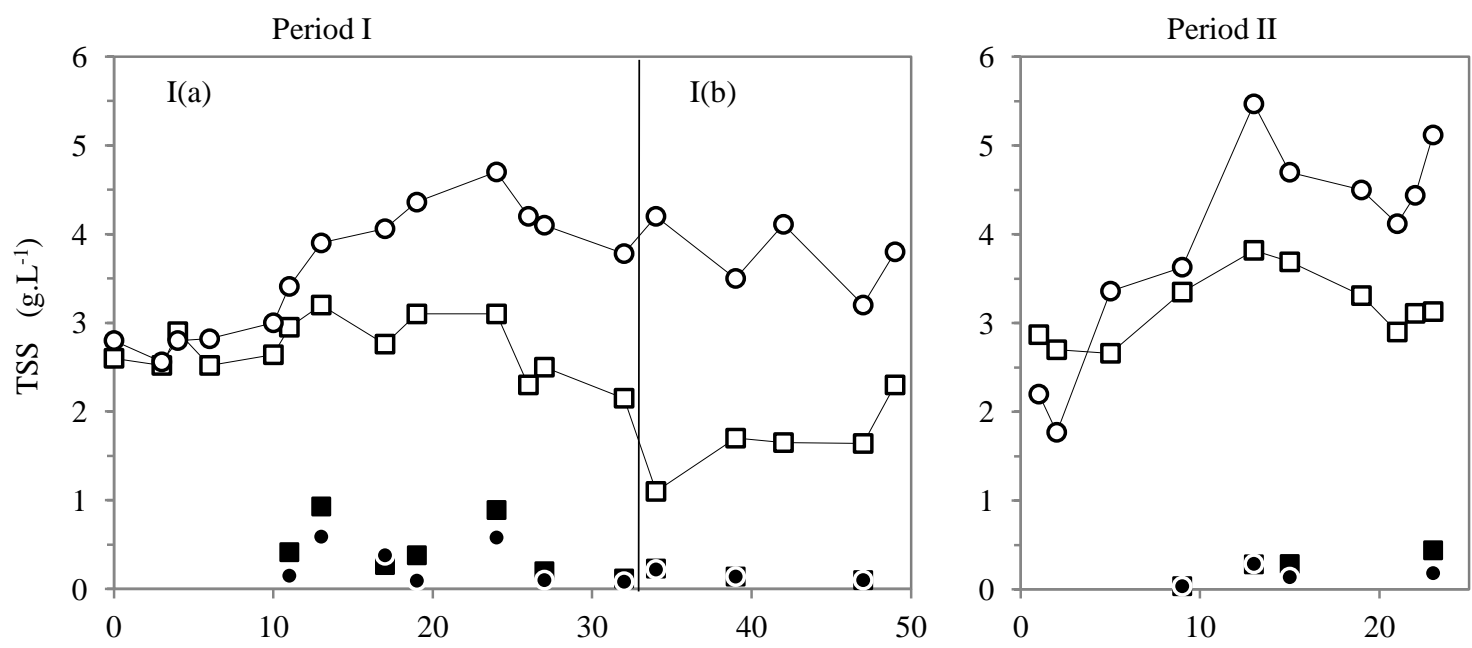

Operational day (day)

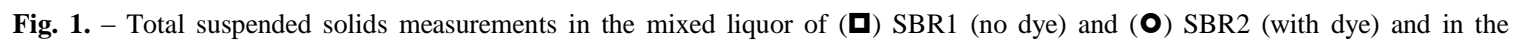

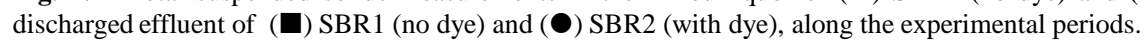




\section{FIGURE 2}

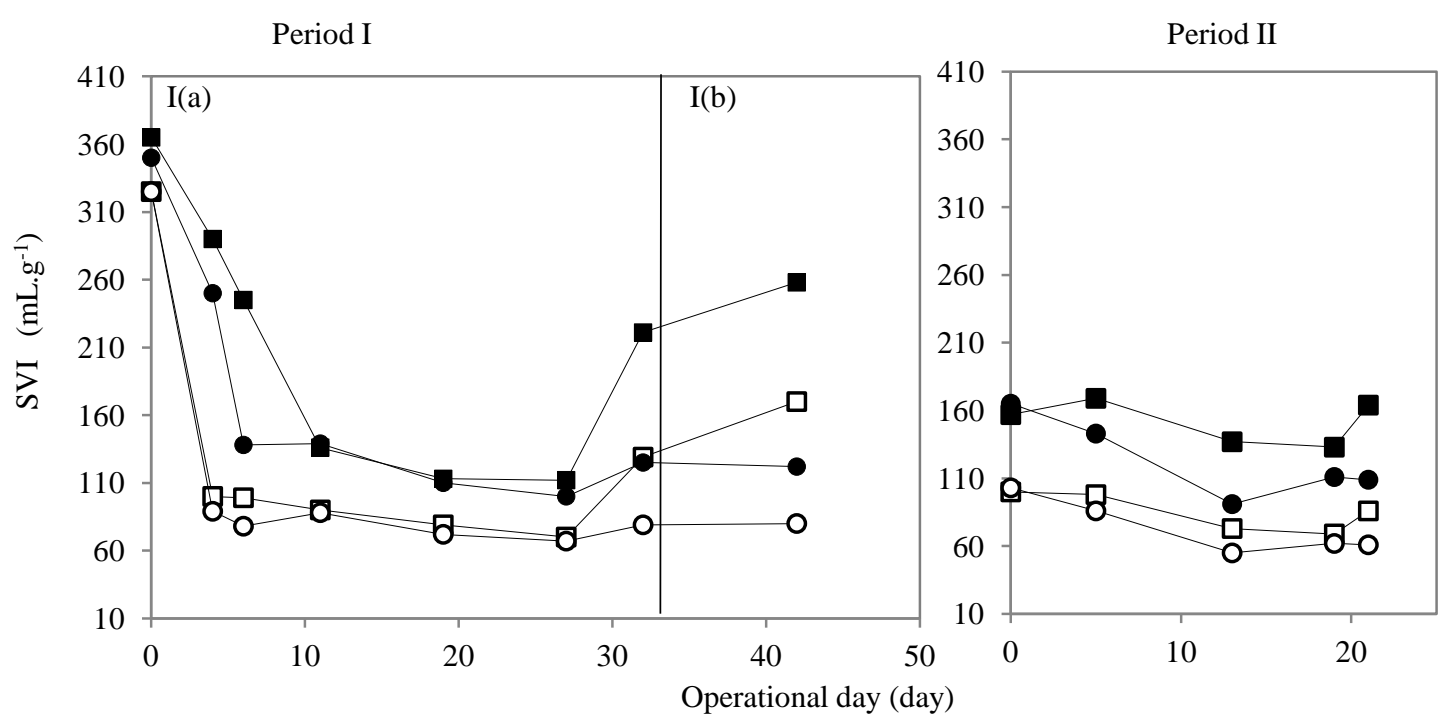

Fig. 2. - Sludge volume index values measured along the experimental periods I and II, namely, $\mathrm{SVI}_{30}$ in (吕) SBR1 (no dye) and (O) SBR2 (with dye), and $\mathrm{SVI}_{5}$ in (ם) SBR1 (no dye) and (-) SBR2 (with dye). 


\section{FIGURE 3}

\section{Flocculent inoculum}

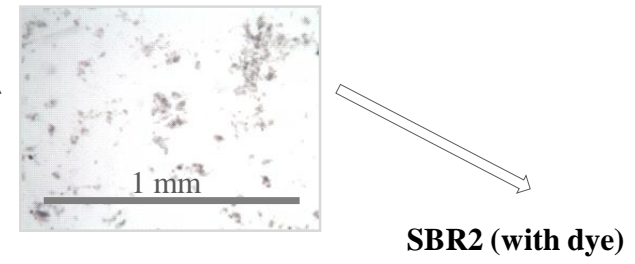

SBR1 (no dye)

(a)

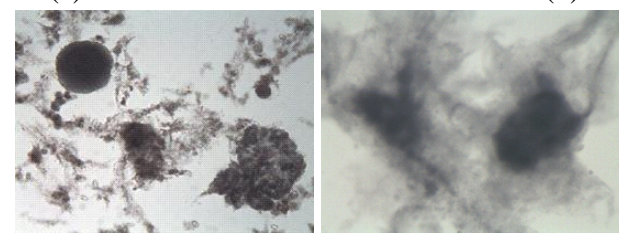

Period II

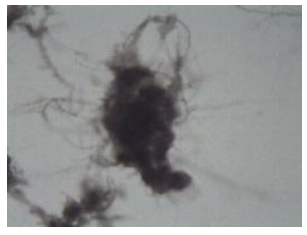

(b)

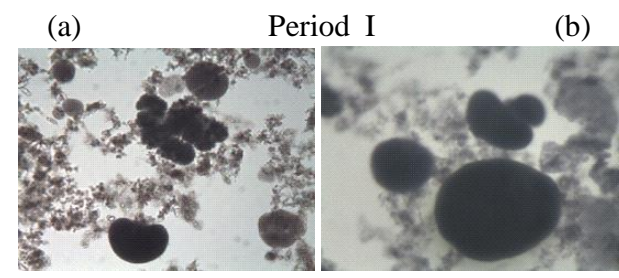

Period II

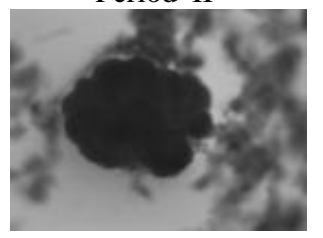

Fig. 3. Microscopy images from biomass samples taken from the activated sludge flocculent inoculum, and from the mixed liquor of the two SBR during period I (Ia - day 21, Ib - day 40) and period II (day 22). 


\section{FIGURE 4}

SBR1 (no dye)
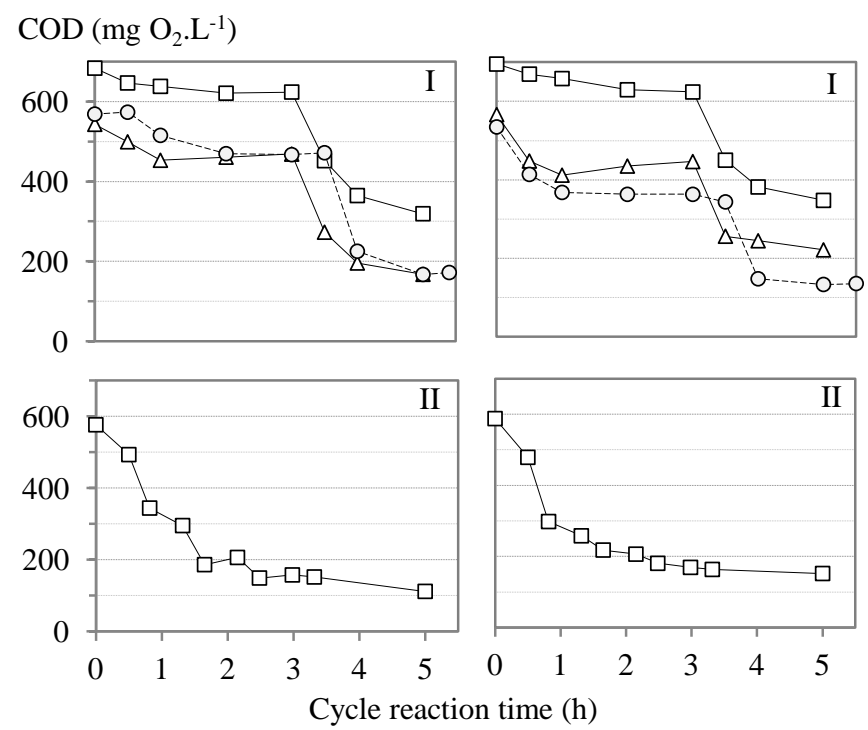

SBR2 (with dye)

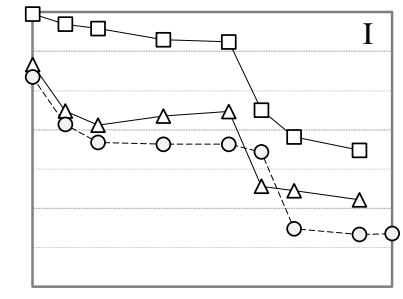

Fig. 4. Representative mixed liquor dissolved COD profiles along the reaction phase of the operational cycle in SBR1 (no dye) and SBR2 (with dye), for each of the experimental periods, I and II. Period I(a): (ם) day 1, $(\triangle)$ day 26; period I(b): ( $\mathbf{O}$, dashed line) day 42; Period II: day 9 . 


\section{FIGURE 5}

Period I

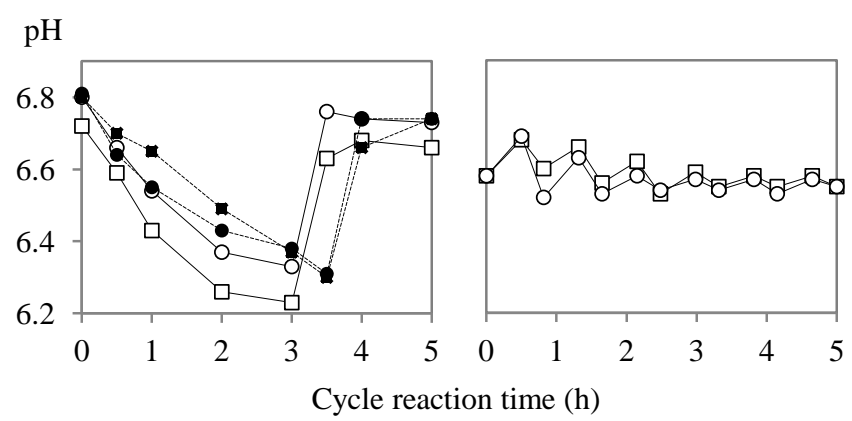

Fig. 5. Representative $\mathrm{pH}$ profiles along the reaction phase of the operational cycle in $(\mathbf{0}, \mathbf{0})$ SBR1 (no dye) and $(\boldsymbol{\square}, \mathbf{\square})$ SBR2 (with

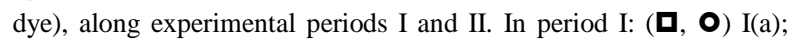
$(\square, 0) \mathrm{I}(\mathrm{b})$. 


\section{FIGURE 6}

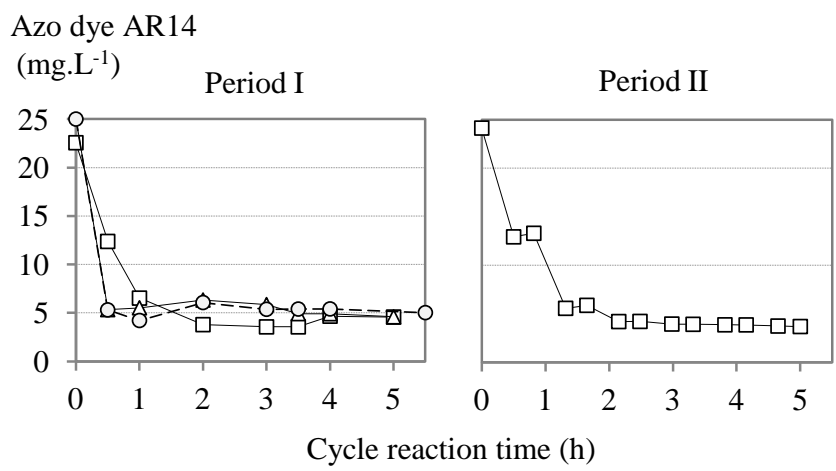

Fig. 6. Representative azo dye concentration profiles (by spectrophotometry) along the reaction phase of the operational cycle in SBR2, for each of the experimental periods, I and II. Period I(a): (口) day $1,(\triangle)$ day 26; period I(b): (O, dashed line) day 49; Period II: day 15 . 


\section{FIGURE 7}

a)

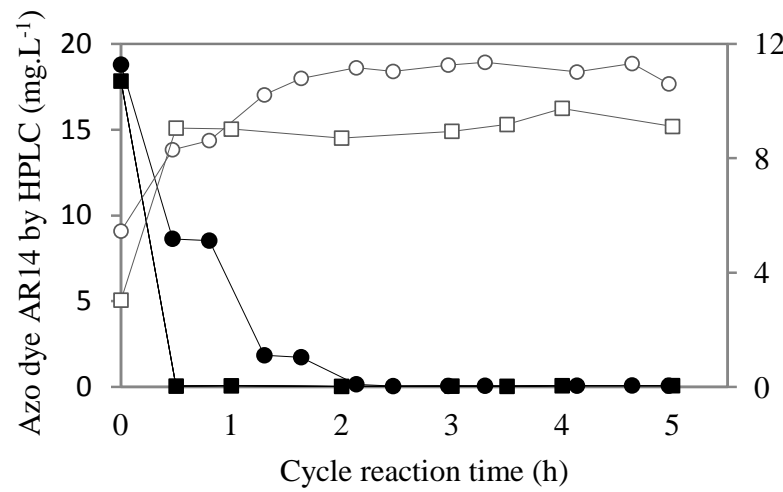

b)

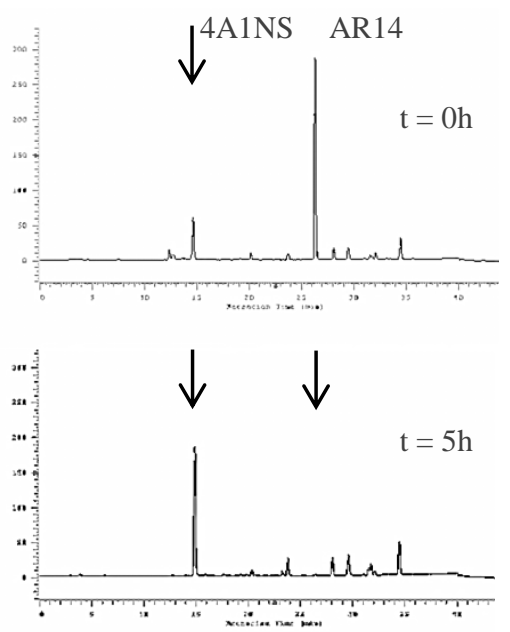

Fig. 7. a) Representative concentration profiles (by HPLC) of ( $\mathbf{\square}, \mathbf{0})$ azo dye AR14 and ( $\mathbf{\square}, \mathbf{0})$ amine 4A1NS, along the reaction phase of SBR2 for each of the experimental periods: $(\boldsymbol{\square}, \mathbf{\square})$ period I; $(\boldsymbol{\bullet}, \mathbf{0})$ Period II. b) Examples of HPLC chromatograms of samples taken during the reaction phase of one of the cycles in period Ia (same scale on both chromatograms). 


\section{FIGURE 8}

SBR1 (no dye)

$\mathrm{ORP}(\mathrm{mV}, \mathrm{Ag} / \mathrm{AgCl})$
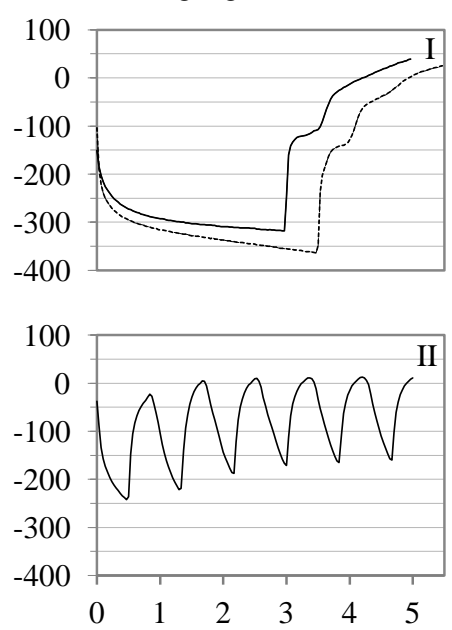

SBR2 (with dye)
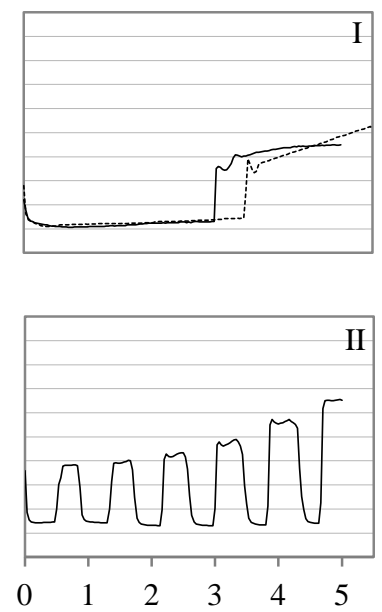

Cycle reaction time $(\mathrm{h})$

Fig. 8. Representative ORP profiles along the reaction phase of the operational cycle in SBR1 (no dye) and SBR2 (with dye), for each of the experimental periods, I and II. Period I: I(a), straight line; I(b), dashed line. 


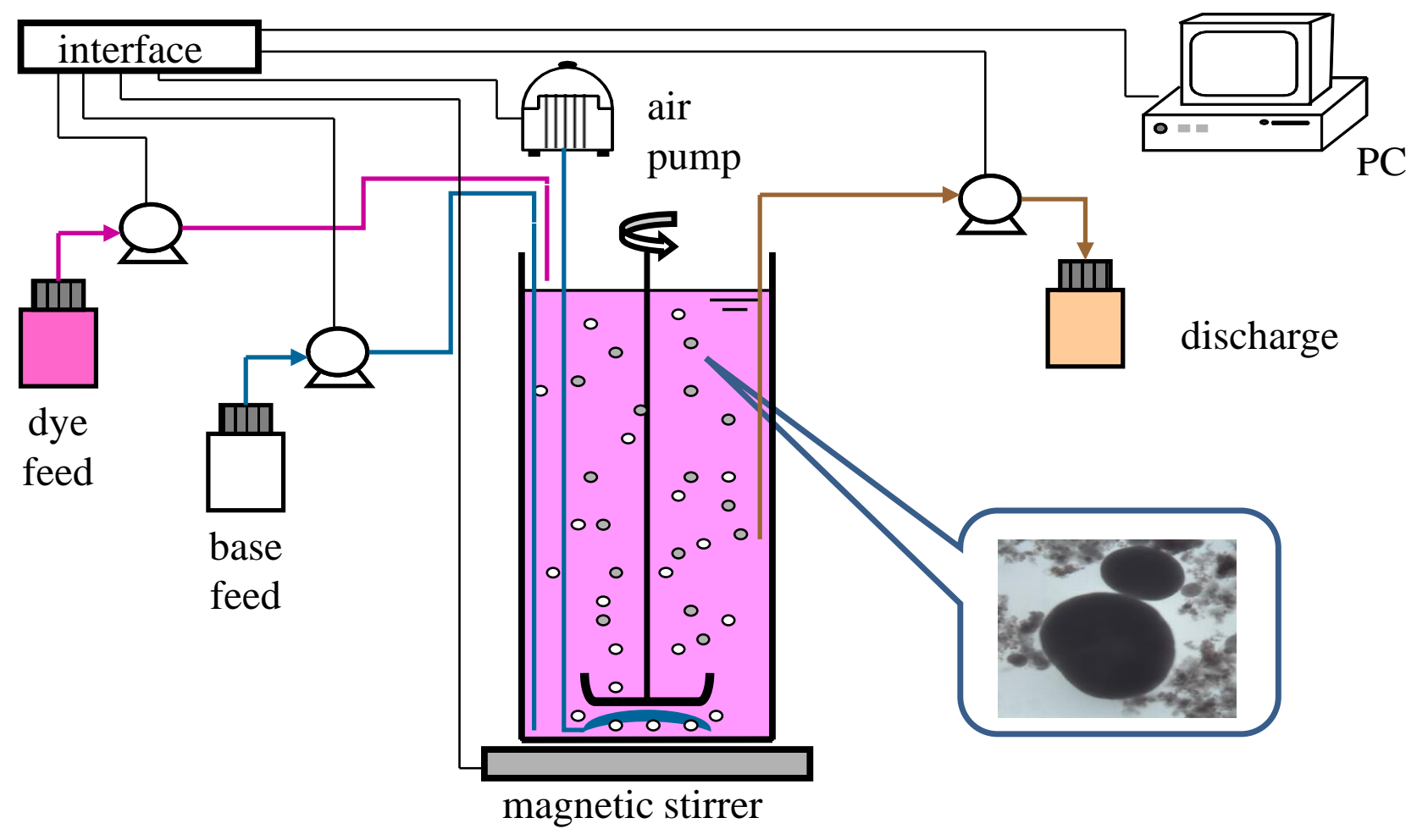

